\title{
KPIs for Optimal Location of charging stations for Electric Vehicles: the Biella case-study
}

\author{
Edoardo Fadda, Daniele Manerba, Gianpiero Cabodi, Paolo Camurati, Roberto Tadei \\ Department of Control and Computer Engineering, Politecnico di Torino \\ Corso Duca degli Abruzzi, 24 - I-10129 Turin, Italy. \\ Email: \{edoardo.fadda, daniele.manerba, gianpiero.cabodi, paolo.camurati, roberto.tadei\}@ polito.it
}

\begin{abstract}
Electric vehicles are accelerating the world's transition to sustainable energy. Nevertheless, the lack of a proper charging station infrastructure in many real implementations still represents an obstacle for the spread of such a technology. In this paper, we present a real case application of optimization techniques in order to solve the location problem of electric charging stations in the district of Biella, Italy. The plan is composed by several progressive installations and decision makers pursue several objectives that might be in contrast. For this reason, we present an innovative framework based on the comparison of several ad-hoc Key Performance Indicators for evaluating many different aspects of a location solution.
\end{abstract}

\section{INTRODUCTION}

Environmental pollution is one of the biggest problems affecting human society, and one of the main source of pollution is represented by motorized vehicles. It has been estimated that they are responsible for $40 \%$ of carbon dioxide emissions and $70 \%$ of other GHG emissions in urban areas [1]. In order to reduce this kind of pollution, an alternative and promising mobility solution is represented by the adoption of electric vehicles (EVs). Nevertheless, the expansion of this technology is strictly linked with the growth of a proper infrastructure for recharging the vehicles.

In this context, the company Ener.bit S.r.l. ${ }^{1}$ and the Department of Control and Computer Engineering of Politecnico di Torino have recently developed a project for the sustainability of electric mobility in the district of Biella, Piedmont (Italy). The project goal was to plan the type, number, and location of the charging stations over an horizon of about 10 years (20192030). It is worthwhile noticing that the number of stations to locate and the number of power plugs for each station depend on an economical analysis related to the forecast number of EVs. Instead, the type of charging stations mainly depends on the features of a selected location. For example, a charging station near working centers can have a low charging system (because workers are assumed to park their vehicle during the entire day), while a charging station near shopping centers is supposed to be faster (cars must be recharged during shopping time). Therefore, the actual decision problem faced in the

\footnotetext{
This work has been supported by Ener.bit S.r.l. (Biella, Italy) under the grants "Studio di fattibilità per la realizzazione di una rete per la mobilità elettrica nella provincia di Biella" and "Analisi per la realizzazione di una rete per la mobilità elettrica nella provincia di Biella".

${ }^{1}$ Official website: http://www.enerbit.it/, last accessed: 2019-04-30.
}

project was to select the municipalities in the Biella district where to locate at least one charging station.

In general, location problems consider several different (and possibly conflicting) objectives, e.g., achieving a level of service proportional to the importance of the location, reducing the worst-case service level, maximizing the average service level, etc. Considering all those objectives in the same mathematical problem may end up with a huge amount of solutions that can confuse the decision maker instead of providing help. For this reason, our study provides an innovative analysis based on the comparison of several different aspects of a location solution through the use of a battery of Key Performance Indicators (KPIs). Moreover, since charging infrastructures are commonly supposed to be located through several progressive interventions over a defined time-horizon, we also analyze the trend of the provided KPIs over the interventions to generate long-term managerial insights.

\section{A. Literature review}

Optimal location is a standard topic in operations research. There is a huge amount of different models, and the choice of the most correct model to abstract the problem depends on the objectives set and the constraints imposed by the application itself. In our case, it is fundamental to provide a constraint on the exact number of municipalities where to locate a charging station. Furthermore, the model should aim at optimizing some quality-of-service metrics for the user community.

In the literature, several works are present in this context. In [1], the authors present a study on the location of charging stations for EVs for the city of Lisbon (Portugal), characterized by a strong concentration of population and movements. The methodology is based on a model that maximizes demand coverage while maintaining an acceptable level of service. In [2], instead, the authors uses a bilevel model in order to optimize vehicle sharing systems.

After a careful study of the existing approaches, and considering the specific features of the application at hand and the requests by the involved company, we decided to analyze the $p$-centdian model, which represents a combination of the classical $p$-center and $p$-median problems [3].

The rest of this paper is organized as follows. Section II is devoted to present the location model used in the project. In Section III, we propose and discuss several different KPIs of 
interest for our application. In Section IV, we describe more in details the project and we present the numerical results. Finally, conclusions are drawn in Section V.

\section{THE P-CENTDIAN MODEL}

Throughout the paper we use the following notation:

- $G=(N, E)$ : complete undirected graph with a set of nodes $N$ representing possible locations for the charging stations and a set of edges $E=\{(i, j) \mid i, j \in N, i \leq j\}$;

- $d_{i j}$ : distance between node $i$ and node $j \in N$ (note that distance $d_{i i}$ may be non-null since it represents the internal distance to travel within municipality $i \in N$ );

- $Q_{i}$ : service demand in node $i \in N$;

- $h_{i}=Q_{i} / \sum_{j \in N} Q_{j}$ : demand rate of node $i \in N$;

- $p$ : predefined number of stations to locate, with $p \leq|N|$;

- $\bar{d}$ : coverage radius, i.e. the threshold distance to discriminate the covering. It represents, e.g., the maximum distance that an EV can travel (due to the battery capacity) or that a user is willing to drive to reach a charging station;

- $\mathcal{C}_{i}=\left\{j \in N, d_{i j} \leq \bar{d}\right\}$ : covering set of $i \in N$, i.e. the set of all stations nearer than $\bar{d}$ from node $i$.

The $p$-centdian problem is to find $p$ nodes where to locate charging stations so as to minimize a linear combination among the maximum and the average (weighted) distance between the located stations and the demand nodes. Its formulation is:

$$
\min \lambda M+(1-\lambda) \sum_{i \in N} h_{i} \sum_{j \in N \mid(i, j) \in E} d_{i j} x_{i j}
$$

subject to

$$
\begin{gathered}
M \geq \sum_{j \in N \mid(i, j) \in E} h_{i} d_{i j} x_{i j} \quad \forall i \in N \\
\sum_{j \in N \mid(i, j) \in E} x_{i j}=1 \quad \forall i \in N \\
\sum_{j \in N} y_{j}=p \\
\sum_{i \in N \mid(i, j) \in E} x_{i j} \leq|N| y_{j} \quad \forall j \in N
\end{gathered}
$$

where $y_{j}$ is a binary variable taking value 1 iff a station is located in node $j \in N$, and 0 otherwise, while $x_{i j}$ is a binary variable taking value 1 iff the demand of node $i \in N$ is served by a charging station located in $j \in N$, and 0 otherwise.

The objective function (1) consists of a linear combination of two terms. The first is the auxiliary variable $M$ that, according to constraints (2), takes the maximum value of the expression $\sum_{j \in N} h_{i} d_{i j} x_{i j}$ over all nodes $i \in N$. In other words, it is the maximum distance between a demand node and its closest station. The second is the average distance traveled by the total demand flow towards charging stations. Clearly, through the parameter $0 \leq \lambda \leq 1$ it is possible to define the relative importance of one objective with respect to the other one. In this work, we set the $\lambda$ parameter dynamically by using the ratio between the optima of the relative p-center and $\mathrm{p}$ median subproblems. In this way we ensure that the two terms of (1) are comparable. Constraints (3) ensure that each demand node is served by exactly one station. Constraint (4) ensures to locate exactly $p$ stations. Finally, logical constraints (5) ensure to locate a station in $j$ (i.e., $y_{j}=1$ ) only if it is assigned to serve at least one demand node (i.e., $\sum_{i \in N} x_{i j}>0$ ).

\section{Key PERFORMANCE INDICATORS}

In this section, we define the set of KPIs that were used in the project in order to measure the performance of the solution provided by the model. For simplicity, we define $\mathcal{L}_{i}=\{j \in$ $\left.\mathcal{C}_{i} \mid y_{j}=1\right\}$ as the set of nodes where a charging station has been located that covers demand node $i$, and $\mathcal{C}=\{i \in$ $N \mid \exists j \in \mathcal{C}_{i}$ such that $\left.y_{j}=1\right\}$ as the set of demand nodes covered by at least one charging station.

The following proposed KPIs consider topological, coverage, and accessibility measures:

\section{- WORST-CASE DISTANCE:}

$$
D_{\max }:=\max _{i \in N} \min _{j \in \mathcal{L}} d_{i j}
$$

represents the maximum distance between a demand node and its closest charging station.

- BEST-CASE DISTANCE:

$$
D_{\min }:=\min _{i \in N} \min _{j \in \mathcal{L}} d_{i j}
$$

represents the minimum distance between a demand node and its closest charging station.

- AVERAGE DISTANCE:

$$
D_{\text {avg }}:=\frac{1}{|N|} \sum_{i \in N} \min _{j \in \mathcal{L}} d_{i j}
$$

represents the average distance between a demand node and its closest charging station.

- DISPERSION.

$$
\text { Disp }:=\sum_{i \in \mathcal{L}} \sum_{j \in \mathcal{L}} d_{i j}
$$

represents the sum of the distances between all the located stations. It is a measure of homogeneity of the service from a purely topological point of view.

- ACCESSIBILITY:

$$
A c c:=\sum_{i \in N} h_{i} A_{i}
$$

is the total accessibility of the charging service, where

$$
A_{i}:=\sum_{j \in \mathcal{L}} e^{-\beta d_{i j}}
$$

is the accessibility of a facility in the sense of [4]. The parameter $\beta>0$ must be calibrated and represents the dispersion of the alternatives in the choice process (the calibration has been performed according to [5] and [6]).

- COVERAGE:

$$
C:=100 *|\mathcal{C}| /|N|
$$


represents, in percentage, the number of covered locations with respect to the total.

- WORST-CASE COVERAGE:

$$
C_{\text {min }}:=\min _{i \in N}\left|\mathcal{L}_{i}\right|
$$

represents the minimum number of charging stations covering a demand node.

- BEST-CASE COVERAGE:

$$
C_{\text {max }}:=\max _{i \in N}\left|\mathcal{L}_{i}\right|
$$

represents the maximum number of charging stations covering a demand node.

- AVERAGE COVERAGE:

$$
C_{a v g}:=\frac{1}{N} \sum_{i \in N}\left|\mathcal{L}_{i}\right|
$$

represents the average number of charging stations covering a demand node.

\section{The Biella CASE-STUdY}

In the aforementioned project, the possible locations are the 78 municipalities of the district of Biella, Italy. From a preceding economical analysis, the company is supposed to install charging stations in one municipality by the end of 2019 , in 10 municipalities by the end of 2022, in 37 by the end of 2025, and in all remaining municipalities by the end of 2030. Moreover, the company assumed a coverage radius $\bar{d}=25$, i.e., a municipality is covered if its distance from the nearest charging station is less than 25 kilometers. We remark that each station may have different size, number of plugs, and capacity in terms of charging. However, as already stated in the Introduction, we just focus on selecting the municipalities of Biella district where to locate at least one charging station, while the real characteristics of the stations will be derived in a successive phase. For example, the number of plugs for each municipality can be calculated as a proportion to the demand rate of that particular municipality (and its surroundings).

The p-centdian model, accurately instantiated with the data deriving from the Biella district case study, can be easily solved by exact algorithms as the branch-and-cut implemented in the available commercial and academic solvers. In our particular case, we used the GUROBI solver v.8.1.0. The resolution was performed on a common PC (Intel Core i75500U CPU@2.40 GHz with 8 GB RAM) and took on average 12 seconds. Notice how the resolution efficiency obtained allows to possibly perform a large number of experiments with different input data, thus refining the analysis.

The solutions for the different time thresholds studied, obtained using the p-centdian model, are the following (clearly, at each intervention, the locations chosen in the previous steps are forced to remain in the solution):

- one municipality ( $p=1$ ) by the end of 2019: the only municipality chosen is Biella, the chief town (see Figure 1). This was expected since Biella is the most important city in terms of demand.
- 10 municipalities $(p=10)$ by the end of 2022: some small municipalities close to and other big ones far from Biella are chosen (see Figure 2).

- 37 municipalities $(p=37)$ by the end of 2025: the solution tends to select municipalities close to the previously selected ones, creating clusters (see Figure 3)

- all municipalities $(p=78)$ by the end of 2030 (this corresponds to the trivial solution with $\left.y_{i}=1, \forall i \in N\right)$.

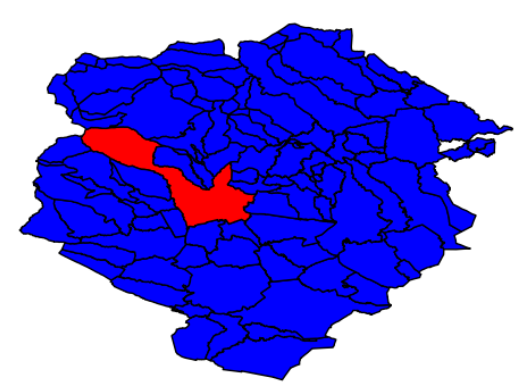

Fig. 1. Optimal location for $p=1$ (2019). Chosen locations in red.

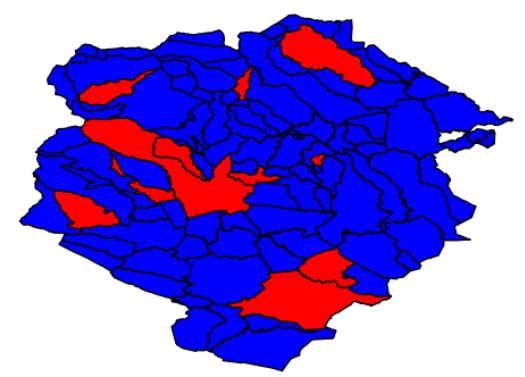

Fig. 2. Optimal location for $p=10$ (2022). Chosen locations in red.

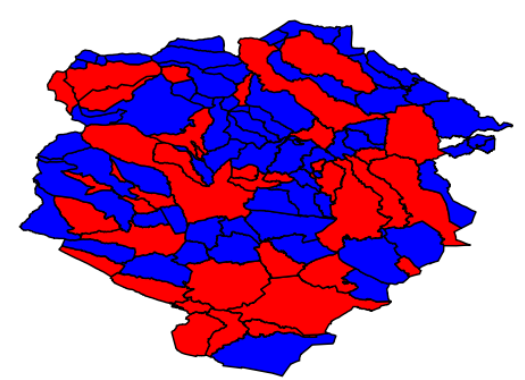

Fig. 3. Optimal location for $p=37$ (2025). Chosen locations in red.

The value of all the KPIs, in the various steps of intervention, is calculated and shown in Table I. Note that the last column, corresponding to the case in which all the locations are chosen, contains the best possible value for each KPI. Several observations can be done: 
- $D_{\max }$ decreases with the increase in the number of municipalities in which at least one charging station has been located and, as it can be seen, it reaches reasonable values from $p=10$ onward.

- $D_{\min }$ decreases as the number of municipalities in which at least one charging station has been located increases, and it stabilizes at the best value already with $p=10$.

- $D_{\text {avg }}$ decreases as the number of municipalities in which at least one charging station has been located increases. It is interesting to note that the percentage improvement in the indicator decreases as the number of selected municipalities increases.

- Disp increases as the number of municipalities in which at least one charging station has been located increases. Its growth is very marked due to the factorial growth of the number of pairs of selected municipalities. The starting value is set to zero since with a single municipality the summation in the definition cannot be calculated.

- Acc increases as the number of municipalities in which at least one charging station has been located increases. Also in this case the improvements are less marked as the number of selected municipalities increases.

- $C$ increases as the number of municipalities in which at least one charging station has been located increases. It can be seen that with only 10 selected municipalities, the coverage reaches very high levels (96\% of the municipalities are covered).

- $C_{m i n}$ increases with the number of municipalities where at least one charging station has been located. Since this is the most pessimistic case, this indicator remains at zero when 1, 10, and 37 selected municipalities are considered. The data then verifies the non-total coverage shown by the KPI previously discussed.

- $C_{\max }$ increases as the number of municipalities in which at least one charging station has been located increases. It can be seen that the increase in value grows with the number of selected municipalities. However, it can be noted that already with 10 municipalities the most covered municipality has the choice between 7 charging stations within a 25 kilometers radius.

- $C_{a v g}$ increases with the increase in the number of municipalities in which at least one charging station has been located and, as it can be seen, has a much lower value than the $C_{\max }$. This implies a heterogeneous situation in terms of coverage of the various locations. In fact, we have a large number of municipalities covered by a few charging stations and a small number of municipalities covered by many charging stations. Since the towns that are not covered are those with a lower demand (i.e., with less electric vehicles) this feature is in line with the technical specifications of the problem.

A common trend of almost all the KPIs is that the second intervention is the one providing the highest proportional change with respect to the previous one (e.g., $C$ almost doubles its value for $p=10$ while it gains only few units for $p=37$
TABLE I

KPIS VALUE IN THE FOUR INTERVENTIONS.

\begin{tabular}{|l|c|c|c|c|}
\hline KPI & $p=1 \mathbf{( 2 0 1 9 )}$ & $p=10 \mathbf{( 2 0 2 2 )}$ & $p=37 \mathbf{( 2 0 2 5 )}$ & $p=78$ (2030) \\
\hline \hline$D_{\max }$ & 53 & 24 & 20 & 11 \\
\hline$D_{\min }$ & 5.7 & 2 & 2 & 2 \\
\hline$D_{\text {avg }}$ & 20.3 & 8.9 & 5.8 & 4.4 \\
\hline isp & 0 & 2158.2 & 34663.9 & 167201.3 \\
\hline$A c c$ & 0.024769 & 0.115986 & 0.329689 & 0.456748 \\
\hline$C$ & $55 \%$ & $96 \%$ & $98 \%$ & $100 \%$ \\
\hline$C_{\min }$ & 0 & 0 & 0 & 1 \\
\hline$C_{\max }$ & 1 & 7 & 22 & 43 \\
\hline$C_{\text {avg }}$ & 0.089744 & 2.653846 & 8.833333 & 19.28205 \\
\hline
\end{tabular}

and $p=78$ ). Interesting enough, $D_{\min }$ reaches its optimal value even for $p=10$. This represents a very important insight for the company for two main reasons. First, it means that the users will perceive the biggest improvement in terms of service in relatively small amount of time (the first 3-5 years) and in response to a small effort in terms of installed stations. Second, it means that the last interventions, which are the ones affected by the most uncertainty (e.g., in terms of economical sustainability), are not very critical for the process overall quality.

\section{CONCLUSIONS}

The implementation of the plan resulting from this study in the district of Biella still needs a detailed urban planning and electrical plant analysis to determine the physical points within the municipalities in which to locate the charging stations identified. However, the described methodologies represent the application of state-of-the-art technology in optimal location to real problems. It is worthwhile noting that the developed analysis can be applied to different location models and to a broader set of KPIs. This way the decision maker can evaluate different solutions and generate insights for the location problem at hand.

\section{REFERENCES}

[1] I. Frade, A. Ribeiro, G. Goncalves, and A. Pais Antunes, "Optimal location of charging stations for electric vehicles in a neighborhood in lisbon, portugal," Transportation Research Record: Journal of the Transportation Research Board, vol. 2252, pp. 91-98, 2011.

[2] A. Quiliot and A. Sarbinowski, "Facility location models for vehicle sharing systems," Proceedings of the 2016 Federated Conference on Computer Science and Information Systems, vol. 8, pp. 605âĂŞ-608, 2016.

[3] H. A. Eiselt and C. L. Sandblom, Decision Analysis, Location Models and Scheduling Problems. Springer-Verlag Berlin Heidelberg, 2004.

[4] W. Hansen, "How accessibility shapes land use," Journal of the American Institute of Planners, vol. 25, pp. 73-76, 1959.

[5] R. Tadei, N. Ricciardi, and G. Perboli, "The stochastic p-median problem with unknown cost probability distribution," Operations Research Letters, vol. 37, pp. 135-141, 2009.

[6] E. Fadda, L. Fotio Tiotsop, D. Manerba, and R. Tadei, "The stochastic multi-path traveling salesman problem with dependent random travel costs," Transportation Science (submitted), 2019. 